\title{
Hepatocellular carcinoma treatment: hurdles, advances and prospects
}

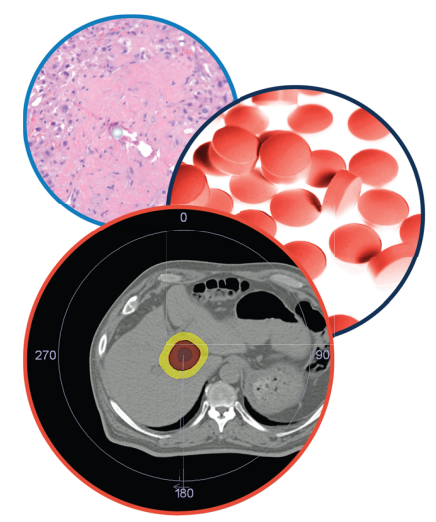
\author{
Behera $^{2}$ \\ ${ }^{1}$ KIIT School of Biotechnology, KIIT University, Bhubaneswar, India \\ ${ }^{2}$ Department of Gastroenterology \& Hepatobiliary Sciences, IMS \& SUM Hospital, Bhubaneswar, India \\ *Author for correspondence: Tel.: +91 8280197 165; ratnaintouch@gmail.com
}

Ratna Kumari*,1, Manoj Kumar Sahu², Anindita Tripathy', Kanishka Uthansingh² \& Manas

\section{Practice points}

- There are two major treatment options for hepatocellular carcinoma (HCC) belonging to different stages (as recommended by the Barcelona Clinic Liver Cancer staging system): curative and palliative treatments.

- Curative treatments like hepatic resection, liver transplantation (LT) and embolizing therapies are recommended for early stage/stage A patients.

- Hepatic resection should be utilized for patients of very early/early stage HCC with uni-focal lesions and well-preserved liver function.

- LT is recommended for patients who meet the Milan criteria and are suitable for hepatic resection.

- Patients waiting for LT should undergo regular screening in order to check if there is any sign of extra-hepatic spread and vascular invasion. Patients beyond the Milan criteria can be considered under expanded criteria and downstaging criteria, but the use of these two criteria in clinical practice has not achieved any consensus.

- Local ablative therapies like radiofrequency ablation and percutaneous ethanol injection are considered for those early stage patients who are not suitable for resection or when LT cannot be done. Radiofrequency ablation can be used as a bridge to LT.

- Palliative therapies are recommended for patients who belong to either intermediate or advanced stage HCC and includes - transarterial chemoembolization (TACE), transarterial radioembolization (TARE), systemic therapies, and molecular targeted therapies.

- Embolizing therapies (TACE, transarterial radioembolization) are recommended for patients classified as the Barcelona Clinic Liver Cancer B/C HCC with inadequate liver function. Drug-eluting bead-TACE has been associated with considerable tumor response and progression-free survival in stage B and C HCC patients.

- Systemic chemotherapies like tamoxifen, cisplatin, and herbal drugs are not recommended for HCC.

- Many molecularly targeted therapies has shown promise in the treatment of advanced stage HCC like sorafenib. It is recommended for stage $C$ patients or patients whose cancer progresses even after locoregional therapies like TACE.

Hepatocellularcarcinoma (HCC) is one of the major causes of cancer-related mortality and is particularly refractory to the available chemotherapeutic drugs. Among various etiologies of HCC, viral etiology is the most common, and, along with alcoholic liver disease and nonalcoholic steatohepatitis, accounts for almost $90 \%$ of all HCC cases. HCC is a heterogeneous tumor associated with multiple signaling pathway alterations and its complex patho-physiology has made the treatment decision challenging. The potential curative treatment options are effective only in small group of patients, while palliative treatments are associated with improved survival and quality of life for intermediate/advanced stage HCC patients. This review article focuses on the currently available treatment strategies and hurdles encountered for HCC therapy. The curative treatment options discussed are surgical resection, liver transplantation, and local ablative therapies which are effective for early stage HCC patients. The palliative treatment options discussed are embolizing therapies, systemic therapies, and molecular targeted therapies. Besides, the review also focuses on hurdles to be conquered for successful treatment of $\mathrm{HCC}$ and specifies the future prospects for HCC treatment. It also discusses the multi-modal approach for HCC management which maximizes the chances of better clinical outcome after treatment and identifies that selection of a particular treatment regimen based on patients' disease stage, patients' ages, and other underlying factors will certainly lead to a better prognosis.

First draft submitted: 19 January 2018; Accepted for publication: 25 June 2018; Published online: 28 September 2018 
Keywords: anti-virals $\bullet$ cell-therapies $\bullet$ curative treatment $\bullet$ Hepatitis $\bullet$ hepatocellular cracinoma $\bullet$ palliative treatment • regorafenib • sorafenib • transarterial chemoembolization • xenotransplantaion

Hepatocellular carcinoma (HCC) is one of the major causes of cancer-related mortality and is particularly refractory to the available chemotherapeutic drugs [1]. In almost all cases HCC is associated with chronic liver damage caused by either viral infections or by other means. Among the diverse etiologies of HCC, viral etiology is the most common particularly infection with Hepatitis B virus. Viral infections along with alcoholic liver diseases and nonalcoholic steatohepatitis (NASH) are major risk factors for developing cirrhosis which forms a background for HCC. The chronic liver diseases and cirrhosis associated HCC accounts for about $90 \%$ of all HCC cases [2]. In most cases, HCC is diagnosed at late stages which limit the treatment option and the currently available therapies are effective only in small group of patients. The complex patho-physiology of HCC has made the treatment decision challenging. There are various guidelines which are published and updated regularly across the world regarding good clinical practice for HCC treatment. The different guidelines across eastern and western countries have similarities as well differences in terms of surveillance and treatment allocation [3]. These differences are mainly due to the differences in the etiologies of HCC and the available resources for HCC treatment, nevertheless the purpose of all these guidelines is to direct the healthcare professionals toward treatment decision-making and improving the treatment outcome.

The available treatment options for HCC patients: advances achieved \& hurdles conquered Depending upon the stage of HCC and extent of underlying liver function abnormalities, there are several treatment regimens (Figure 1). Basically, two major types of treatment strategies are followed - curative and palliative. Potentially curative treatments for very early and early stage HCC patients include surgical resection, liver transplantation and percutaneous ablation. However, for intermediate and advanced stage patients palliative treatment options are preferred like transarterial chemoembolization (TACE), radioembolization, and systemic therapies [4]. There are pros and cons associated with all treatment modalities available.

\section{Curative treatments}

Surgical resection (hepatectomy)

Suitable for very early and early stage HCC, when the liver is in a good functional state and has adequate hepatic reserves. It is the particular treatment of choice for non-cirrhotic liver and patients with uni-focal lesions. The 5 -year survival rates for patients with significant portal hypertension and high bilirubin levels are only $25 \%$, while in patients without portal hypertension and normal bilirubin levels it is 75\% [5]. In noncirrhotic patients hepatectomy can be performed as major hepatectomy to remove a big tumor, whereas in cirrhotic liver or in benign liver tumors, it is done mostly as partial hepatectomy [6]. Few studies report major hepatectomies for large HCC ( $>10 \mathrm{~cm}$ in diameter) with low morbidity and mortality [7-9]. Several studies have also reported the low mortality, tolerable morbidity, and encouraging survival benefits of surgical resection in patients with advanced stage HCC [10]. The critical hurdle in surgical resection of cirrhotic liver is the presence of inadequate hepatic reserves and occurrence of perioperative death. The surgical resection is not preferred in cirrhotic patients when there is presence of multiple tumors, extra-hepatic masses, involvement of main bile duct, and presence of portal hypertension [11], even though portal hypertension is considered as the most important predictor of poor outcome in cirrhotic HCC patients [12]. There should be careful preoperative evaluation of liver for the symptoms associated with portal hypertension, which is crucial for the determination of tumor(s) relation to major vasculature of liver [13]. For cirrhotic patients, there is a strict requirement of minimal remnant liver volume after surgical resection which should be approximately $40 \%$ [14], in contrast to patients without cirrhosis which should be more or less $20 \%$ [11] to avoid postoperative liver failure. The surgical resection is usually curative with improved short-term survival but is associated with high recurrence rate and postoperative death. The rate of recurrence varies between 40 and $80 \%$ within 5 years of surgical resection [15] which is particularly dependent on underlying cirrhosis and active hepatitis [16]. Patients with early recurrence are recommended to have palliative therapies like TACE, radioembolization, and systemic therapies while patients with late recurrence could benefit from repeat resection [17]. 


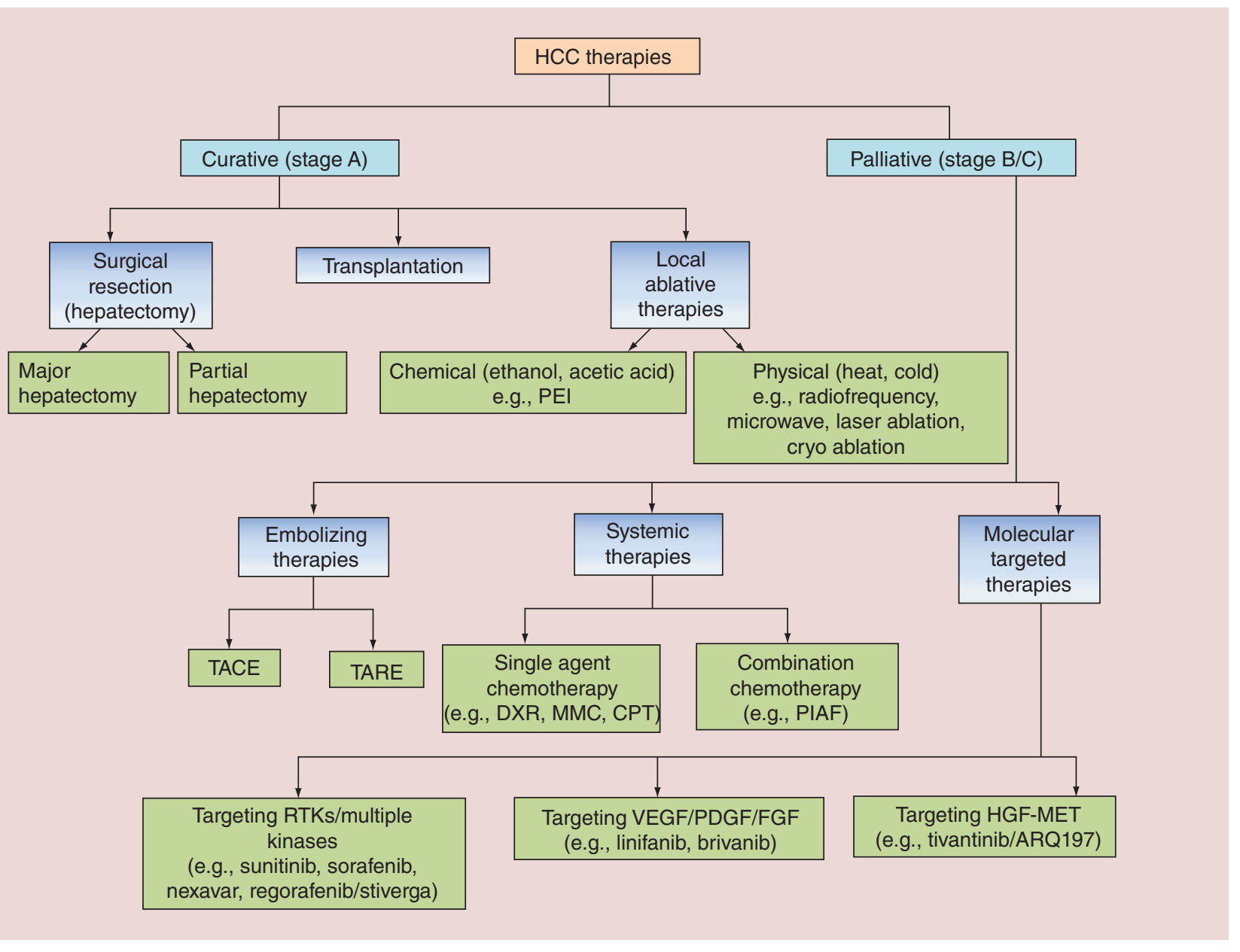

Figure 1. Classification of available hepatocellular carcinoma therapies based on the stages of patients. Curative therapies are suitable for early stage patients and includes surgical resection, liver transplantation, and local ablative therapies. Physical ablation can be by done any energy source, which works by heating (radiofrequency, microwave, and laser) or cooling (cryo/freeze-thaw) of tumor affected area. Palliative therapies includes embolizing, systemic and molecular targeted therapies.

CPT: Cisplatin; DXR: Doxorubicin; HCC: Hepatocellular carcionoma; MMC: Mitomycin C; PEI: Percutaneous ethanol injection; PIAF:

Cisplatin, interferon, adriamycin, and fluorouracil.

Liver transplantation

This is the best treatment option for cirrhotic HCC patients; it not only removes the tumor lesion but also rectifies the underlying hepatic insufficiency, in this way decreasing the rate of recurrence [18]. Patients are referred for LT if they meet Milan criteria $(\mathrm{MiC})$, which states LT can be performed if the liver has one tumor less than $5 \mathrm{~cm}$ or three tumors less than $3 \mathrm{~cm}$ each, without extra-hepatic spread [19]. Meeting MiC before LT reduces the recurrence rate of HCC to less than 15\% and increases 5-year overall response rate (ORR) to 68\% [20]. Besides, MiC, which is totally dependent on the size and number of tumor(s), there have been recent studies demonstrating the role of molecular marker alpha fetoprotein (AFP) and response to loco-regional therapy under the selection criteria of LT [21].

One of the biggest obstacles in LT is that the demand for liver grafts exceeds the supply of liver grafts. It is well known that many patients who meet MiC for LT need to wait because of teh shortage of liver grafts, and by the time the liver graft is available they become unsuitable for LT. In most cases, these patients are found to be invariably associated with extra-hepatic spread and vascular-invasion that disqualifies them for LT [22]. In these cases transplantation can be considered using two criteria - expanded criteria when HCC exceeds MiC during the presentation without any prior treatment and downstaging criteria under which advanced HCC is downstaged through different loco-regional therapies to fulfill the MiC for LT [23]. Single-center studies have demonstrated LT with expanded criteria could achieve post-transplantation benefit comparable with that of LT 
within MiC [24]. Downstaging of tumors before LT with several loco-regional therapies is proposed for patients who could achieve post-transplantation benefits similar to those of LT within MiC. TACE is the major treatment strategy for downstaging; however, radioembolization has also been demonstrated to be a promising strategy for downstaging [25]. However, the use of these two criteria in clinical practice has not achieved any consensus [25]. Downstaging criteria have shown encouraging results in single-centered studies [26] and in multicentered studies [27] with standardized downstaging protocols. However, more single and multicentered studies are needed to make downstaging criteria for $\mathrm{LT}$ beyond $\mathrm{MiC}$, a standard procedure.

Other than HCC qualifying MiC, the indications for LT also include hepatitis so a scoring system - model for end-stage liver disease (MELD) - which is a good predictor of early mortality in cirrhosis patients and in patients with end-stage liver disease [28,29], was used within MiC for deceased donor liver allocation in HCC patients. However, MELD could not predict mortality in HCC patients for which MELD exception was developed based on the tumor burden [30].

Local ablative therapies

Ablative therapies are the best treatment option for patients not suitable for surgical resection or LT. There are two basic types - chemical ablation (ethanol and acetic acid), and physical ablation by using different energy sources (heat and cold). Application of local ablative therapies usually does not cause any systemic side effect. Percutaneous ethanol injection (PEI) and radiofrequency ablation (RFA) are the most widely used ablative therapies [31]. Several studies have shown that RFA is able to achieve complete tumor removal in more than $90 \%$ of HCC cases with a single tumor of less than $4 \mathrm{~cm}[32,33]$. It is extensively used to treat small metastatic HCC [34]. RFA is largely preferred over PEI as it increases survival of HCC patients by 3-years as compared with PEI shown by a recent meta-analysis [35]. While in some cases where tumors are adjacent to large blood vessels in which case RFA cannot be performed, PEI is preferred [32]. The disease-free survival rate is lower for ablative therapies that is due to both higher AFP levels and cirrhosis [36]. These therapies are also known to be associated with high recurrence rate when compared with surgical resection as found in a retrospective analysis [37] and are preferred only when the patient is not a surgical candidate. Another recent meta-analysis of 23 studies by Feng $e$ t al. demonstrated that for treatment of small HCC, the surgical resection resulted in better outcome in terms of ORR and recurrence-free overall survival (OS) as compared to RFA [38].

\section{Palliative treatment}

The curative interventions are limited to a small group of patients because of various internal and external factors like tumor-size, multi-focality, metastasic nature, liver function status, organ shortage, and late diagnosis. All of these reasons can lead to an inclination toward palliative therapies. Palliative therapies are preferred for patients classified as Barcelona Clinic Liver Cancer (BCLC) stage B and C and aim toward escalating survival or improving quality of life for patients.

\section{Embolizing therapies for HCC}

Embolization is the process to obstruct the blood flow to cancer cells. HCC is largely dependent on arterial blood supply $(75-80 \%)$ in contrast to normal liver, so it is logical to embolize hepatic arterial supply in HCC to induce tumor-hypoxia and necrosis. Embolization can be done directly with only embolic agents or in combination with chemotherapy/radiotherapy.

\section{Transarterial chemoembolization}

TACE is recommended for patients classified as BCLC stage B/C HCC with compensated liver function. It is also increasingly recommended for patients under the waiting list for LT in order to prevent further tumor progression [39]. TACE is a technique where subsequent to chemotherapy directly into the hepatic artery, embolization is done. The embolizing agents can be of two types - the first type that can embolize temporarily like gelatin sponge, autologous blood clot and the second type that can embolize permanently like polyvinyl alcohol, steel coils, and microspheres. The gelatin sponge is the most extensively used embolizing agent [40]. When TACE is performed without any drug, it is called transarterial embolization (TAE), and is done to embolize arterial supply only. An advancement over TACE is DEB-TACE (drug eluting bead-TACE) that uses embolizing particles, which also acts as a carrier for the chemotherapeutic drug. The purpose is to embolize the hepatic artery and to provide a slow and steady release of chemotherapeutic drug which increases the exposure of tumor cells to chemotherapeutic drugs. 
DEB-TACE has been associated with considerable tumor response and progression-free survival in stage B and C HCC patients with acceptable toxicity [41].

TACE is the treatment of choice for patients with multi-focal large lesions without any vascular-invasion and extra-hepatic spread as recommended by EASL [12]. TACE is well tolerated with less than $5 \%$ of cases of complications [42]. Postembolization syndrome is the most common complications occurring after TACE but can be controlled by analgesics and antiemetics [43]. Although there is no standardized protocol and consensus for the use of a chemotherapeutic agent for TACE, but the use of doxorubicin and cisplatin is most common $[44,45]$.

\section{Transarterial radioembolization}

Transarterial radioembolization (TARE) is relatively a new therapeutic option for BCLC stage B/C HCC and involves selective intra-arterial administration of Yittrium (Y90) loaded microspheres or lipidol labeled with rhenium or iodine [46]. Two types of microspheres are used currently for TARE, these are glass-made theraspheres and resin-made SIR spheres with different embolic powers and activities. TARE removes the chances of hepatic ischemia after delivering local radiation to liver tumors and is preferred for the treatment of HCC patients with portal vein thrombosis [47].

Microsphere brachytherapy is a useful approach for the treatment of intermediate and advanced stage HCC when curative treatment is not advisable. Brachytherapy permits selective radiation source to be implanted within the tumor thereby increasing selectivity and avoiding the exposure of normal hepatic cells to the harmful radiations. Embolization of hepatic arteries with appropriately calibrated microspheres combined with a high energy radiation source is done. A high energy $\beta$-emitter Y90 loaded in glass or resin microspheres are used in the process to embolize the hepatic artery and this technique is evaluated in a number of clinical trials while few studies have shown that radioembolization is associated with better response and fewer side effects when compared with TACE [48]. A few multicentre trials comparing TARE with sorafenib have been reported, in which some are completed, such as SORAMIC [49] and SARAH [50], while some are still ongoing [51] and recruiting patients [52]. SORAMIC evaluated Y90-loaded microsphere therapy followed by sorafenib and demonstrated that it showed a similar level of toxicity as sorafenib alone [49]. On the other hand, SARAH compared TARE treatment with sorafenib in advanced HCC patients [50].

Systemic therapies for HCC

Systemic therapies with chemotherapeutic drugs had long been tried in advanced HCC without showing significant progress in the outcome of HCC. Chemotherapeutic agents like cisplatin, doxorubicin, 5-fluorouracil and gemcitabine are associated with only marginal improvement in survival and the response rate remains low [53]. There are a few combination chemotherapy regimens, among which PIAF (cisplatin, interferon, adriamycin, and fluorouracil) has shown promise in a Phase II clinical study [54], but failed to show efficacy when compared with doxorubicin alone in a Phase III clinical study [55]. Moreover, the patients showed severe myelotoxicity to the PIAF regimen [55]. As in most cases, HCC is diagnosed in the advanced stage so additional systemic agents, combination chemotherapy, and molecular targeted agents are immediately needed for a better therapeutic outcome.

Molecular targeted therapies for HCC

The recent advances in the knowledge of deregulated molecular pathways involved in the development of HCC have led to the identification of novel targeted therapies. The altered molecular pathways, which have been well described and are involved in the pathogenesis of HCC, could be exploited for HCC chemoprevention. Some of the molecular pathways deregulated in HCC are: Wnt/B-catenin; c-Met; EGFR/RAS/MAPK; Hedgehog; VEGF/PDGF; PI3K/AKT/mTOR; and IGF signaling pathways [56. Many drugs modulating these pathways are already in Phase II and III clinical trials. Some of the targeted agents under clinical development are the following:

\section{Sunitinib for targeting several RTKs}

The VEGF pathway has been found to be involved in a variety of cancers and particularly play a significant role in HCC pathogenesis [57]. Sunitinib is a tyrosine kinase inhibitor with antiangiogenic and antitumorigenic activity targeting VEGF-R, PDGF-R and other RTKs. Several clinical studies to check the antitumor efficacy of sunitinib in advanced HCC patients has been done. The reports showed persistent toxicity, low response rate, and modest antitumor activity after sunitinib treatment in advanced HCC patients [58]. 


\section{Linifanib/ABT-869 for targeting VEGF/PDGF pathways}

Linifanib is a novel ATP competitive inhibitor mainly active against all VEGF-R and PDGF-R but show very mild activity against other RTKs and serine-threonine kinases [59]. Phase II trial with linifanib in patients with unresectable and metastatic HCC showed a positive outcome. The objective response rate with single agent linifanib was $9.1 \%$ and OS was 9.7 months with manageable adverse events [60] which demonstrates that linifanib is clinically active with an acceptable safety profile in advanced HCC patients.

\section{Brivanib for targeting VEGF/FGF pathway}

Brivanib is a dual tyrosine kinase inhibitor that selectively targets VEGF-R and FGF-R tyrosine kinases [61]. Brivanib showed an antiangiogenic effect in various tumors including liver [62]. Brivanib showed potential antitumor activity considering improvements in the secondary outcome of HCC treatment with a frequently associated adverse effect of hyponatremia [62]. This study warrants further investigation as the secondary outcome measures are based on unvalidated mRECIST (modified response evaluation criteria in solid tumors) criteria for HCC.

\section{Sorafenib/nexavar for targeting multiple kinases}

Sorafenib is a multikinase inhibitor with efficacy against VEGF and PDGF receptor tyrosine kinases and the downstream serine/threonine kinases Ras/Raf. All of them are actively involved in tumor proliferation and angiogenesis [63]. On the basis of Phase II and Phase III trials, sorafenib is the only approved drug for unresectable HCC treatment [64]. Two randomized double-blind, multicenter Phase III trials were done to check the efficacy of sorafenib monotherapy in advanced HCC patients - The AP(Asia-Pacific) and SHARP(Sorafenib HCC Assessment Randomized Protocol) trials. Both trials demonstrated sorafenib is more efficacious than any other treatment for unresectable HCC. They demonstrated improved survival, increased time to radiologic progression and time to symptomatic progression [63]. The 1-year survival rates were $44 \%$ in sorafenib monotherapy compared with placebo treatment (33\%) in the SHARP trial. On the other hand, the 6-month survival rates in the AP trial were 53 and $37 \%$ for the sorafenib monotherapy and placebo treatment groups, respectively [63].

Sorafenib has also been used in combination with other therapies, and various single-center and multicenter clinical trials have been reported for sorafenib in combination with other treatment modalities. These interventions included TACE [65], radioembolization [66], chemotherapeutic drugs [67] and molecular targeted therapies [68-70]. Unfortunately, most of these trials had a significant problem in study design and conduct with the majority of these studies showed no advantage in combination therapy compared with the sorafenib alone.

\section{Tivantinib/ARQ 197 for targeting HGF-MET axis}

The HGF-MET pathway is involved in tumor growth and metastatic progression. Inhibiting MET with tivantinib in in vitro and in vivo models has shown to induce apoptosis and block growth [71]. In a randomized clinical trial in HCC, rivantinib demonstrated significant improvement in OS only in MET-high patients and time to progression (TTP) was more in the tivantinib treatment (1.6 months) group as compared with the placebo treatment (1.4 months) group [72]. Tivantinib also demonstrated efficacy in treating MET high patients who did not get benefit after first-line sorafenib treatment and the disease progressed [73]. Based on the promising results with Tivantinib, a Phase III trial has been initiated in MET high HCC patients, who are intolerant to sorafenib and have a progressive disease. The trial is still ongoing [74].

\section{Regorafenib/Stiverga targeting multiple kinases}

Regorafenib is recently approved by the FDA as a second-line treatment for HCC patients, who failed to respond to sorafenib. Both regorafenib and sorafenib block multiple tyrosine kinases but regorafenib has stronger activity against VEGF receptors and c-KIT. Regorafenib is also shown to partially block TIE2 angiopoietin receptor that plays an important role in angiogenesis [75], which makes regorafenib a potentially strong inhibitor of angiogenesis than sorafenib [76]. The RESORCE (regorafenib after sorafenib in patients with hepatocellular carcinoma) trial demonstrated that regorafenib treatment improved OS and TTP of HCC patients (median survival: 10.6 months; TTP: 3.2 months) as compared with placebo treatment (median survival: 7.8 months; TTP: 1.5 months) group [76].

\section{Hurdles to be conquered}

The treatment strategies discussed above have their own limitations due to several medical and psycho-social reasons. Hurdles in HCC treatment occurs at various levels including at the level of screening, diagnosis, treatment, and at 


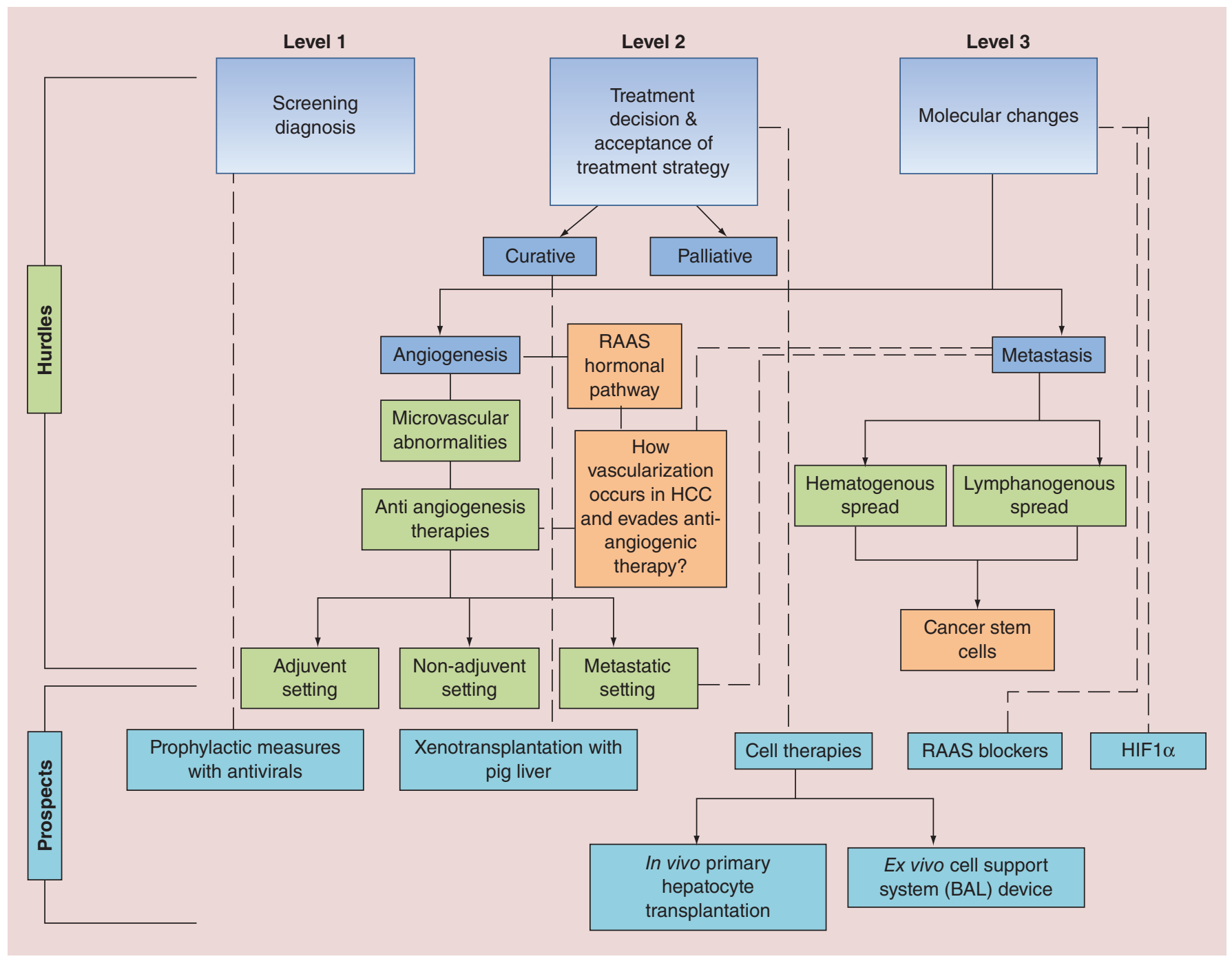

Figure 2. Hurdles in hepatocellular carcinoma treatment classified as level 1, level 2 and level 3 . Level 1 includes hurdles encountered at screening and diagnosis which involves asymptomatic disease, poor awareness of patient, lack of resources, as in case of low middle income countries, and patient's inability to pay for the tests. Educational hurdles include both poor patient's education and insufficient knowledge of healthcare provider. Level 2 includes treatment decision and acceptance of the treatment strategies and involves picking right treatment strategy based on the stage and liver function status. Level 3 includes hurdles in hepatocellular carcinoma (HCC) treatment at molecular level which holds potential to investigate various molecular changes observed in HCC patients. All these hurdles show the way to several prospects for HCC treatment which include prophylactic measures, xenotransplantion with pig liver, cell therapies and targeting awry molecules/pathways. The dotted lines in the flowchart depicts correlated aspects of hurdles and prospects. BAL: Bio-artificial liver; HCC: Hepatocellular carcionoma; PHT: Primary hepatocyte transplantation; RAAS: Renin-angiotensin-aldosterone system.

the level of acceptance of the proposed solution for HCC (Figure 2). The majority of efforts have been made to conquer hurdles at the level of screening, diagnosis, and treatment of HCC with considerable success. However, a lot more needs to be done to conquer the hurdles in HCC treatment at a molecular level.

One of the significant hurdles in current cancer treatment at the molecular level is angiogenesis, which is an important requirement for tumor growth. As HCC is a highly vascular cancer characterized by microvascular abnormalities, which could be reversed by antiangiogenic therapies [77]. But the problem exists in determining the efficacy of these therapies which can be done by functional vascular imaging like - dynamic contrast enhancedMRI (DCE-MRI), CT-perfusion (DCE-CT), DCE-ultrasonography and functional PET. However, they tend to be relatively nonspecific for vascular imaging and are semi-quantitative. For better characterization of tumor angiogenesis, a more targeted approach is required like a targeted PET, or SPECT based imaging [78] is required. 
Since sorafenib works by inhibiting the VEGF-R pathway, demonstrating the potential of antiangiogenic therapies for HCC, the redundant and complex angiogenic pathways must be explored for a better therapeutic outcome. Despite the acceptance of sorafenib as a life-prolonging drug for advanced HCC patients, its efficacy is very much dependent on the etiologies of HCC, as is evident by some discrepancies in AP and SHARP trials. So it is conceivable to explore other antiangiogenic therapies and also the combination of sorafenib with other treatment modalities, which is already under active investigation.

Since angiogenesis is also affected by angiotensin II(AT II) and insulin resistance (IR) so targeting these pathways also holds great antiangiogenic potential [79]. RAAS (renin-angiotensin-aldosteron system) blockers inhibit the AT II/AT I-receptor axis, which plays an important role in tumor neovascularization [80]. Moreover, a preclinical study reported the suppression of hepatocarcinogenesis by the inhibition of aldosterone, [81] which is a downstream mediator of AT II. Therefore, it is conceivable to explore other antiangiogenic therapies and also the combination of these therapies with other treatment modalities, which is already under active investigation.

Metastasis is another most difficult hurdle for the successful treatment of most cancers including HCC; consequently, it accounts for most cancer suffering and deaths. The intrahepatic metastasis of HCC is more common compared with the extrahepatic metastasis, which is a rare event at the time of diagnosis [82]. In most cases, intrahepatic metastasis can be treated successfully with various locoregional therapies even on a recurrent lesion, but it is difficult to treat extra-hepatic metastasis and is considered as a terminal event in HCC [83]. Although it is generally accepted that angiogenesis and hematogenous metastasis is the most common route for solid tumor growth and spread, however, the recent studies indicate that lymphangiogenesis and lymphanogenous spread is another important way for metastasis [84]. As hepatic lymphangeogenesis is largely unknown, so it is worth exploring the role of signaling mediators of lymphangeogenesis in HCC progression which can be a potential therapeutic target for HCC. There are significant amount of data available which suggests that cancer stem cells (CSCs) have great potential for angiogenesis and lymphangeogenesis. CSCs express angiogenic/lymphangeogenic factors during tumorigenesis and are known to transdifferentiate into endothelial cells, thus playing a major role in tumor angiogenesis/vasculogenesis [84] and holding potential to be a therapeutic target.

\section{Prospects: multi-modal approach for HCC management}

In order to achieve maximum benefit for HCC treatment, the multi-modal approach should be opted for, depending upon the stage of HCC and functional liver reserves. Several clinical trials are ongoing to make out the best treatment course for HCC patients, which would be potent, well tolerated and provide improved quality of life. Some of the new prospect for HCC treatment with great potential are discussed below:

\section{Prophylactic measures with antivirals}

Sustained viremia and associated inflammation in the liver can lead to hepatocarcinogenesis, so the use of antivirals can prevent HCC occurrence/recurrence by reducing viral load. Antiviral therapies include oral nucleoside and nucleotide analogues(NAs)-like lamivudine(LAM). The advantages of antivirals include relatively rare adverse effects and easy administration through oral route. Lamivudine (LAM) is one of the antivirals that has been studied most for its effect on HBV associated HCC. Several studies have demonstrated that LAM significantly decreased the incidence of HCC [86].

Direct-acting antivirals (DAA) is another group of antivirals that is currently under clinical trials for the treatment of HCV linked HCC. The use of DAA in HCV infected patients has led to the cure of a significantly increased number of cirrhotic patient but they must go for regular surveillance [87].

\section{Xenotranplantation of liver}

Xenotransplantation with pig liver is an exciting area of research and has been tried in patients with acute liver failure to overcome organ shortage. One of the major concerns for xenotransplantation is graft rejection as the immune response to xenografts is stronger in comparison with allografts. Making humanized/transgenic pigs through genetic engineering approach is the possible solution to outwit rejection, while it can also be done by increasing the immune tolerance of the recipient [88]. 


\section{Cell therapies}

One of the alternatives emerged for LT is cell therapy that included both stem cells and somatic cells. Primary hepatocytes are the first type of cells through which cell therapies were performed. Broadly two basic types of cell therapies have been evaluated for their potential in treating the liver disease are the following:

In vivo primary hepatocyte transplantation

Primary hepatocyte transplantaion (PHT) has been initially used to treat patients with liver-based metabolic disorders and acute liver failure as a substitute/bridge to LT. A significant number of patients have undergone PHT for various liver-based metabolic disorders [89]. Although the hepatocyte function starts deteriorating after 1 year of transplantation, but their clinical outcome demonstrated obvious improvement. Poor engraftment of hepatocytes and immediate cell loss due to host immune response are two major causes for decline in hepatocyte function. To overcome these barriers, the transplantation of encapsulated hepatocytes in alginate beads has been tried, which resulted in better engraftment, cell survival and decreased host immune response. It is also speculated that coencapsulating mesenchymal stem cells with hepatocytes would improve hepatocyte function [90].

Ex vivo cell support system/bio-artificial liver (BAL) devices

The ex vivo support system for liver was first evaluated by Eiseman et al. (1965) in a patient of reversible hepatic failure with an isolated perfused heterologous liver [91]. The first BAL device used ex vivo suspension of porcine hepatocytes in a bioreactor; the treatment duration of this device was only for few hours because of the death of hepatocytes in suspension [92] and was later overcome by use of hepatocyte spheroids [93].

\section{Hypoxia \& hypoxia-inducible factor $1 \alpha$}

Hypoxia, or insufficient oxygen supply, to the core of solid tumor is itself harmful to the tumors, but it can induce several pro-survival pathways that contribute to angiogenesis and tumor proliferation. The regulation of various prosurvival pathways by HIF- $1 \alpha$ makes it an attractive therapeutic target for cancer. HIF- $1 \alpha$ is known to be over-expressed in HCC and is associated with poor prognosis; its expression strongly correlates to the clinical outcome in HCC patients [94]. In several preclinical models and hepatoma cell-lines, HIF-1 $\alpha$ has been targeted through the activation of hydroxylases or by using small molecule inhibitor or by the use of a fusion protein in tumor xenograft model. Another way to target HIF-1 $\alpha$ is targeting at the mRNA level, mRNA antagonist of HIF- $1 \alpha$ has been developed and are currently under Phase Ib clinical trial in HCC patients after the first-line treatment. Additionally, the possibility of targeting HIF-1 $\alpha$ in combination with other chemotherapeutic drugs holds a promise for the development of a better systemic therapy for HCC treatment.

\section{Conclusion}

Despite the aggressive and refractory nature of HCC, it can be treated or better managed with a multi-modal approach which includes the active participation of pathologists, oncologists, radiologists, surgeons, and hepatologists. This approach maximizes the chances of better clinical outcome after treatment. Selection of a particular treatment regimen based on patients disease stage, patients age, and other underlying factors will certainly lead to a better prognosis. However, most of the approaches discussed above have their own shortcomings despite that the use of sorafenib, tivantinib, and regorafenib holds a great promise for advanced stage HCC treatment. In addition, a recent expansion in the understanding of HCC molecular pathogenesis and technical advances achieved in this field has empowered the treatment prospects like the use of hepatocyte transplantation instead of whole liver transplantation has enormous potential embedded. Moreover, the use of effective antivirals as a prophylactic measure and targeting a new multi-functional molecule/pathway like HIF-1 $\alpha$, RAAS-pathway blockers can lead to a better prognosis and thus holds a significant potential for HCC treatment.

\section{Future perspective}

The review presents an overview of treatment approaches available for early, intermediate, and advanced stage of HCC patients. Additionally, it summarizes hurdles to be encountered and future prospects for HCC treatment. As screening and surveillance has improved the diagnosis of HCC patients at early stages so curative treatments could be a potential therapeutic option in future for HCC patients. So improving over the barriers of liver transplantation has enormous potential, like use of cell therapies. On the other hand, the treatment of intermediate/advanced stage HCC in future would greatly be affected by targeting potential new molecules like HIf- $1 \alpha$ and RAAS pathway. 
Financial \& competing interests disclosure

The authors have no relevant affiliations or financial involvement with any organization or entity with a financial interest in or financial conflict with the subject matter or materials discussed in the manuscript. This includes employment, consultancies, honoraria, stock ownership or options, expert testimony, grants or patents received or pending, or royalties.

No writing assistance was utilized in the production of this manuscript.

\section{Open access}

This work is licensed under the Attribution-NonCommercial-NoDerivatives 4.0 Unported License. To view a copy of this license, visit http://creativecommons.org/licenses/by-nc-nd/4.0/

\section{References}

Papers of special note have been highlighted as: $\bullet$ of interest; $\bullet \bullet$ of considerable interest

1. Kumari R, Sharma A, Ajay AK, Bhatt M. Mitomycin C induces bystander killing in homogeneous and heterogeneous hepatoma cellular models. Mol. Cancer 8, 87 (2009)

2. Davis GL, Dempster J, Meler JD et al. Hepatocellular carcinoma: management of an increasingly common problem. Proc. (Bayl. Univ. Med. Cent.) 21(3), 266-280 (2008).

3. Yu SJ. A concise review of updated guidelines regarding the management of hepatocellular carcinoma around the world: 2010-2016. Clin. Mol. Hepatol. 22(1), 7-17 (2016).

- Updated guidelines about hepatocellular carcinoma (HCC) management.

4. Gomma AI, Waked I. Recent advances in multidisciplinary management of hepatocellular carcinoma. World J. Hepatol. 7(4), 673-687 (2015).

5. Llovet JM, Fuster J, Bruix J. Intention-to-treat analysis of surgical treatment for early hepatocellular carcinoma: Resection versus transplantation. Hepatology 30(6), 1434-1440 (1999).

6. Xu H, Mao Y. A brief comment on liver resection for hepatocellular carcinoma. Gastroenterol. Rep. (Oxf.) 1(3), 184-185 (2013)

7. Bismuth H, Chiche L, Castaing D. Surgical treatment of hepatocellular carcinomas in noncirrhotic liver: experience with 68 liver resections. World J. Surg. 19, 35-41 (1995).

8. Kawasaki S, Makuuchi M, Miyagawa S et al. Results of hepatic resection for hepatocellular carcinoma. World J. Surg. 19, 31-34 (1995).

9. Vauthey JN, Klimstra D, Franceschi D et al. Hepatic resection for hepatocellular carcinoma. Am. J. Surg. 169, 28-35 (1995).

10. Yang $\mathrm{T}$, Lin $\mathrm{C}$, Zhai J et al. Surgical resection for advanced hepatocellular carcinoma according to Barcelona Clinic Liver Cancer (BCLC) staging. J. Cancer Res. Clin. Oncol. 138(7), 1121-1129 (2012).

- Surgical resection analysis for HCC.

11. Belghiti J, Ogata S. Assessment of hepatic reserve for the indication of hepatic resection. J. Hepatobiliary Pancreat. Surg. 12, 1-3 (2005).

12. European Association For The Study Of The Liver; European Organisation For Research And Treatment Of Cancer. EASL-EORTC clinical practice guidelines: management of hepatocellular carcinoma. J. Hepatol. 56, 908-943 (2012).

-. Clinical practice guidelines for HCC.

13. Cooper A, Aloia TA. Surgical resection for hepatocellular carcinoma. Transl. Cancer Res. 2(6), 450-459 (2013).

14. Kishi Y, Abdalla EK, Chun YS et al. Three hundred and one consecutive extended right hepatectomies: evaluation of outcome based on systematic liver volumetry. Ann. Surg. 250, 540-548 (2009).

15. Portolani N, Coniglio A, Ghidoni S et al. Early and late recurrence after liver resection for hepatocellular carcinoma: prognostic and therapeutic implications. Ann. Surg. 243, 229-235 (2006).

16. Matsuda M, Fujii H, Kono $\mathrm{H}$ et al. Surgical treatment of recurrent hepatocellular carcinoma based on the mode of recurrence: repeat hepatic resection or ablation are good choices for patients with recurrent multicentric cancer. J. Hepatobiliary Pancreat. Surg. 8, 353-359 (2001).

17. Huang ZY, Liang BY, Xiong M et al. Long-term outcomes of repeat hepatic resection in patients with recurrent hepatocellular carcinoma and analysis of recurrent types and their prognosis: a single-center experience in China. Ann. Surg. Oncol. 19, 2515-2525 (2012).

18. Mazzaferro V, Chun YS, Poon RT et al. Liver transplantation for hepatocellular carcinoma. Ann. Surg. Oncol. 15, 1001-1007 (2008).

- Liver transplantation guidelines for HCC.

19. Mazzaferro V, Regalia E, Doci R et al. Liver transplantation for the treatment of small hepatocellular carcinomas in patients with cirrhosis. N. Engl. J. Med. 334, 693-699 (1996).

20. Onaca N, Davis GL, Jennings LW et al. Improved results of transplantation for hepatocellular carcinoma: a report from the international registry of hepatic tumors in liver transplantation. Liver Transpl. 15, 574-580 (2009).

21. Mehta N, Yao FY. Moving past 'one size (and number) fits all' in the selection of candidates with hepatocellular carcinoma for liver transplant. Liver Transpl. 19, 1055-1058 (2013). 
22. Yao FY, Fidelman N. Reassessing the boundaries of liver transplantation for hepatocellular carcinoma: where do we stand with tumor down-staging? Hepatology 63(3), 1014-1025 (2016).

\section{-. Liver transplantation assessment in HCC.}

23. Silva MF, Sherman M. Criteria for liver transplantation for HCC: what should the limits be? J. Hepatol. 55(5), 1137-1147 (2011).

24. Yao FY, Ferrell L, Bass NM et al. Liver transplantation for hepatocellular carcinoma: expansion of the tumor size limits does not adversely impact survival. Hepatology 33, 1394-1403 (2001).

25. Prasad KR, Young RS, Burra P et al. Summary of candidate selection and expanded criteria for liver transplantation for hepatocellular carcinoma: a review and consensus statement. Liver Transpl. 17(Suppl. 2), S81-S89 (2011).

26. Kim Y, Stahl CC, Makramalla A et al. Downstaging therapy followed by liver transplantation for hepatocellular carcinoma beyond Milan criteria. Surgery 162(6), 1250-1258 (2017)

- Milan criteria expansion.

27. Mehta N, Guy J, Frenette CT et al. Excellent outcomes of liver transplantation following down staging of hepatocellular carcinoma to within milan criteria: a multicenter study. Clin. Gastroenterol. Hepatol. 16(6), 955-964 (2018)

28. Malinchoc M, Kamath PS, Gordon FD et al. A model to predict poor survival in patients undergoing transjugular intrahepatic portosystemic shunts. Hepatology 31, 864-871 (2000).

29. Kamath PS, Wiesner RH, Malinchoc $\mathrm{M}$ et al. A model to predict survival in patients with end-stage liver disease. Hepatology 33 , 464-470 (2001).

30. Massie AB, Caffo B, Gentry SE et al. MELD exceptions and rates of waiting list outcomes. Am. J. Transplant. 11, 2362-2371 (2011).

31. Thandassery RB, Goenka U, Goenka MK. Role of local ablative therapy for hepatocellular carcinoma. J. Clin. Exp. Hepatol. 4, S104-S111 (2014).

32. Brunello F, Veltri A, Carucci P et al. Radiofrequency ablation versus ethanol injection for early hepatocellular carcinoma: a randomized controlled trial. Scand. J. Gastroenterol. 43, 727-735 (2008).

33. Lin SM, Lin CJ, Lin CC et al. Radiofrequency ablation improves prognosis compared with ethanol injection for hepatocellular carcinoma $<$ or $=4 \mathrm{~cm}$. Gastroenterology 127, 1714-1723 (2004).

34. Lencioni R, Cioni D, Crocetti L et al. Early-stage hepatocellular carcinoma in patients with cirrhosis: long-term results of percutaneous image-guided radiofrequency ablation. Radiology 234(3), 961-967 (2005).

35. Yang B, Zan RY, Wang SY et al. Radiofrequency ablation versus percutaneous ethanol injection for hepatocellular carcinoma: a meta-analysis of randomized controlled trials. World J. Surg. Oncol. 13, 96 (2015).

- Meta-analysis regarding radiofrequency ablation and percutaneous ethanol injection treatment.

36. Chen MS, Li JQ, Zheng Y et al. A prospective randomized trial comparing percutaneous local ablative therapy and partial hepatectomy for small hepatocellular carcinoma. Ann. Surg. 243, 321-328 (2006).

37. Guglielmi A, Ruzzenente A, Valdegamberi A et al. Radiofrequency ablation versus surgical resection for the treatment of hepatocellular carcinoma in cirrhosis. J. Gastrointest. Surg. 12, 192-198 (2008).

38. Feng Q, Chi Y, Liu Y, Zhang L, Liu Q. Efficacy and safety of percutaneous radiofrequency ablation versus surgical resection for small hepatocellular carcinoma: a meta-analysis of 23 studies. J. Cancer Res. Clin. Oncol. 141(1), 1-9 (2015).

39. Otto G, Schuchmann M, Hoppe-Lotichius M et al. How to decide about liver transplantation in patients with hepatocellular carcinoma: size and number of lesions or response to TACE? J. Hepatol. 59(2), 279-284 (2013).

40. Wáng YXJ, De Baere T, Idée JE, Ballet S. Transcatheter embolization therapy in liver cancer: an update of clinical evidences. Chin. J. Cancer Res. 27(2), 96121 (2015).

41. Luz JH, Luz PM, Martin HS et al. DEB TACE for intermediate and advanced HCC: initial experience in a Brazilian Cancer Center. Cancer Imaging 17(1), 5 (2017)

42. Clark TW. Complications of hepatic chemoembolization. Semin. Intervent. Radiol. 23, 119-125 (2006).

43. Leung DA, Goin JE, Sickles C et al. Determinants of postembolization syndrome after hepatic chemoembolization. J. Vasc. Interv. Radiol. 12, 321-326 (2001).

44. Bruix J, Sherman M. Management of hepatocellular carcinoma: an update. Hepatology 53, 1020-1022 (2011).

\section{- HCC management approaches.}

45. Marelli L, Stigliano R, Triantos C et al. Transarterial therapy for hepatocellular carcinoma: which technique is more effective? A systematic review of cohort and randomized studies. Cardiovasc. Intervent. Radiol. 30, 6-25 (2007).

46. Sacco R, Conte C, Tumino E et al. Transarterial radioembolization for hepatocellular carcinoma: a review. J. Hepatocell. Carcinoma 3 , 25-29 (2016).

47. Cho YY, Lee M, Kim HC et al. Radioembolization is a safe and effective treatment for hepatocellular carcinoma with portal vein thrombosis: a propensity score analysis. PLoS ONE 11(5), e0154986 (2016).

48. Kim HC. Radioembolization for the treatment of hepatocellular carcinoma. Clin. Mol. Hepatol. 23(2), 109-114 (2017). 
49. Ricke J, Bulla K, Kolligs F et al. Safety and toxicity of radioembolization plus sorafenib in advanced hepatocellular carcinoma: analysis of the European multicentre trial SORAMIC. Liver Int. 35(2), 620-626 (2015).

50. Vilgrain V, Abdel-Rehim M, Sibert A et al. Radioembolisation with yttrium90 microspheres versus sorafenib for treatment of advanced hepatocellular carcinoma (SARAH): study protocol for a randomised controlled trial. Trials 15, 474 (2014).

51. Clinical Trials With TheraSphere ${ }^{\circledR}$ YTTRIUM-90 GLASS MICROSPHERES. 2016. www.therasphere.com/clinicaltrials_us/index.asp

52. Clinicaltrials.gov. Study to compare selective internal radiation therapy (SIRT) versus sorafenib in locally advanced hepatocellular carcinoma (HCC) (SIRveNIB). (2014) https://clinicaltrials.gov/ct2/show/study/NCT01135056?term=01135056\&rank=1

53. Lai CL, Wu PC, Chan GC et al. Doxorubicin versus no antitumor therapy in inoperable hepatocellular carcinoma. A no antitumor therapy in inoperable hepatocellular carcinoma. A prospective randomized trial. Cancer 62, 47983 (1988).

54. Leung TW, Patt YZ, Lau WY et al. Complete pathological remission is possible with systemic combination chemotherapy for inoperable hepatocellular carcinoma. Clin. Cancer Res. 5, 1676-1681 (1999).

55. Yeo W, Mok TS, Zee B et al. A randomized Phase III study of doxorubicin versus cisplatin/interferon alpha-2b/doxorubicin/fluorouracil (PIAF) combination chemotherapy for unresectable hepatocellular carcinoma. J. Natl Cancer Inst. 97, 1532-1538 (2005).

56. Moeini A, Cornellà H, Villanueva A. Emerging signaling pathways in hepatocellular carcinoma. Liver Cancer 1(2), 83-93 (2012).

57. Zhang ZL, Liu ZS, Sun Q. Expression of angiopoitins, Tie2 and vascular endothelial growth factor in angiogenesis and progression of hepatocellular carcinoma. World J. Gastroenterol. 12, 4241-4245 (2006).

58. Faivre S, Raymond E, Boucher E et al. Safety and efficacy of sunitinib in patients with advanced hepatocellular carcinoma: an open-label, multicentre, Phase II study. Lancet Oncol. 10, 794-800 (2009).

59. Albert DH, Tapang P, Magoc TJ et al. Preclinical activity of ABT-869, a multitargeted receptor tyrosine kinase inhibitor. Mol. Cancer Ther. 5(4), 995-1006 (2006).

60. Toh HC, Chen PJ, Carr BI et al. Phase 2 trial of linifanib (ABT-869) in patients with unresectable or metastatic hepatocellular carcinoma. Cancer 119(2), 380-387 (2013).

61. Bhide RS, Lombardo LJ, Hunt JT et al. The antiangiogenic activity in xenograft models of brivanib, a dual inhibitor of vascular endothelial growth factor receptor-2 and fibroblast growth factor receptor-1 kinases. Mol. Cancer Ther. 9, 369-378 (2010).

62. Jhonson PJ, Qin S, Park JW et al. Brivanib versus sorafenib as first-line therapy in patients with unresectable, advanced hepatocellular carcinoma: results from the randomized Phase III Brisk-FL study. J. Clin. Oncol. 31(28), 3509-3516 (2013).

63. Keating GM, Santoro A. Sorafenib a review of its use in advanced hepatocellular carcinoma. Drugs 69(2), 223-240 (2009).

- Sorafenib study in HCC.

64. Sorafenib, package insert .Bayer Healthcare Pharmaceuticals, Inc. CA, USA. http://berlex.bayerhealthcare.com/html/products/pi/Nexavar_PI.pdf

65. Pawlik TM, Reyes TK, Cosgrove D et al. Phase II trial of sorafenib combined with concurrent transarterial chemoembolization with drug-eluting beads for hepatocellular carcinoma. J. Clin. Oncol. 29(30), 3960-3967 (2011).

66. Salman A, Simoneau E, Hassanain M et al. Combined sorafenib and yttrium-90 radioembolization for the treatment of advanced hepatocellular carcinoma. Curr. Oncol. 23(5), e472-e480 (2016).

67. Abou-Alfa GK, Johnson P, Knox JJ et al. Doxorubicin plus sorafenib vs doxorubicin alone in patients with advanced hepatocellular carcinoma: a randomized trial. JAMA 304(19), 2154-2160 (2010).

68. Cheng AL, Kang YK, Lin DY et al. Sunitinib versus sorafenib in advanced hepatocellular cancer: results of a randomized Phase III trial. J. Clin. Oncol. 31, 4067-4075 (2013).

69. Cainap C, Qin S, Huang WT et al. Linifanib verses sorafenib in patients with advanced hepatocellular carcinoma: results of a randomized Phase III trial. J. Clin. Oncol. 33, 172-179 (2014).

70. Zhu AX, Rosmorduc O, Evans TRJ et al. SEARCH: A phase III, randomized, double-blind, placebo-controlled trial of sorafenib plus erlotinib in patients with advanced hepatocellular carcinoma. J. Clin. Oncol. 33, 559-566 (2014).

71. Jeay S, Munshi N, Hill J et al. ARQ 197, a highly selective small molecule inhibitor of c-MET, with selective anti-tumor properties in a broad spectrum of human cancer cells. Presented at: AACR Annual Meeting. CA, USA, 14-18 April 2007 (Abstract 2369).

72. Santoro A, Rimassa L, Borbath I et al. Tivantinib for second-line treatment of advanced hepatocellular carcinoma: a randomised, placebo-controlled Phase ii study. Lancet Oncol. 14(1), 55-63 (2013).

73. Martell RE, Puzanov I, Ma WW et al. Safety and efficacy of MET inhibitor tivantinib (ARQ 197) combined with sorafenib in patients (pts) with hepatocellular carcinoma (HCC) from a hepatocellular carcinoma (HCC) from a Phase I study. J. Clin. Oncol. 30(Suppl.), Abstract 4117 (2012).

74. Study of tivantinib in subjects with inoperable hepatocellular carcinoma who have been treated with one prior therapy (METIV HCC). www.clinicaltrials.gov/ct2/show/NCT017557

75. Martin V, Liu D, Fueyo J, Gomez-Manzano C. Tie2: a journey from normal angiogenesis to cancer and beyond. Histol. Histopathol. 23(6), 773-780 (2008). 
76. Frennette CT. The role of regorafenib in hepatocellular carcinoma. Gastroenterol. Hepatol. (N Y). 13(2), 122-124 (2017).

77. Jain RK. Normalization of tumor vasculature: an emerging concept in antiangiogenic therapy. Science 307, 58-62 (2005).

78. Turkbey B, Kobayashi H, Ogawa M, Bernardo M, Choyke PL. Imaging of tumor angiogenesis: functional or targeted? AJR Am. J. Roentgenol. 193(2), 304-313 (2009).

79. Kaji K, Yoshiji H, Ikenaka Y et al. Possible involvement of angiogenesis in chronic liver diseases: interaction among renin-angiotensin-aldosterone system, insulin resistance and oxidative stress. Curr. Med. Chem. 19(12), 1889-1898 (2012).

- The renin-angiotensin-aldosterone system in liver diseases.

80. Huang W, Wu YL, Zhong J et al. Angiotensin II type 1 receptor antagonist suppress angiogenesis and growth of gastric cancer xenografts. Dig. Dis. Sci. 53(5), 1206-1210 (2008).

81. Kaji K, Yoshiji H, Kitade M et al. Selective aldosterone blocker, eplerenone, attenuates hepatocellular carcinoma growth and angiogenesis in mice. Hepatol. Res. 40(5), 540-549 (2010).

82. Bae SY, Kim HJ, Oh HH et al. Multiple bone metastases as the first manifestation of hepatocellular carcinoma in patient with noncirrhotic liver. Case Rep. Oncol. Med. 2015, 512849 (2015).

83. Bruix J, Sherman M. Management of hepatocellular carcinoma. Hepatology 42, 1208-1236 (2005).

84. Li S, Li Q. Cancer stem cells and tumor metastasis (Review). Int. J. Oncol. 4, 1806-1812 (2014)

85. Sie M, den Dunnen WF, Hoving EW et al. Antiangiogenic therapy in pediatric brain tumors: an effective strategy? Crit. Rev. Oncol. Hematol. 89(3), 418-432 (2014).

86. Lai CL, Yuen MF. Prevention of hepatitis B virus-related hepatocellular carcinoma with antiviral therapy. Hepatology 57, 399-408 (2013).

87. Baumert TF, Jühling F, Ono A, Hoshida Y. Hepatitis C-related hepatocellular carcinoma in the era of new generation antivirals. BMC Med. 15(1), 52 (2017)

88. Cooper DKC, Dou KF, Tao KS et al. Pig liver xenotransplantation: a review of progress toward the clinic. Transplantation 100, 2039-2047 (2016).

89. Dhawan A, Puppi J, Hughes RD, Mitry RR. Human hepatocyte transplantation: current experience and future challenges. Nature Rev. Gastroenterol. Hepatol. 7, 288-298 (2010).

90. Dhawan A. Clinical human hepatocyte transplantation: current status and challenges. Liver Transpl. 21, S39-S44 (2015).

- Human hepatocyte transplantation study.

91. Eiseman B, Liem DS, Raffucci F. Heterologous liver perfusion in treatment of hepatic failure. Ann. Surg. 162, 329-345 (1965).

92. Matsumura K, Guevara G, Huston H et al. Hybrid bioartificial liver in hepatic failure: preliminary clinical report. Surgery 101, 99-103 (1987).

93. Sakai Y, Naruse K, Nagashima I et al. Large-scale preparation and function of porcine hepatocyte spheroids. Int. J. Artif. Organs 19(5), 294-301 (1996).

94. Xiang ZL, Zeng ZC, Fan J et al. The expression of HIF-1 $\alpha$ in primary hepatocellular carcinoma and its correlation with radiotherapy response and clinical outcome. Mol. Biol. Rep. 39, 2021-2029 (2012). 
